\title{
On numerical simulation of high-speed CCD/CMOS-based wavefront sensors for adaptive optics
}

\author{
Mikhail V. Konnik and James Stuart Welsh \\ School of Electrical Engineering and Computer Science, \\ University of Newcastle, Callaghan 2308, Australia
}

\begin{abstract}
Wavefront sensors, which use solid-state CCD or CMOS photosensors, are sources of errors in adaptive optic systems. Inaccuracy in the detection of wavefront distortions introduces considerable errors into wavefront reconstruction and leads to overall performance degradation of the adaptive optics system. The accuracy of wavefront sensors is significantly affected by photosensor noise. Thus, it is crucial to formulate high-level photosensor models that enable adaptive optic engineers to simulate realistic effects of noise from wavefront sensors. However, the complexity of solid-state photosensors and multiple noise sources makes it difficult to formulate an adequate model of the photosensor. Moreover, the characterisation of the simulated sensor and comparison with real hardware is often incomplete due to lack of comprehensive standards and guidelines. Owe to these difficulties, engineers work with oversimplified models of the wavefront sensors and consequently have imprecise numerical simulation results.

The paper presents an approach for the modelling of noise sources for CCD and CMOS sensors that are used for wavefront sensing in adaptive optics. Both dark and light noise such as fixed pattern noise, photon shot noise, and read noises, as well as, charge-to-voltage noises are described. Procedures for characterisation of both light and dark noises of the simulated photosensors are provided. Numerical simulation results of a photosensor for a high-frame rate Shack-Hartmann wavefront sensor are presented.
\end{abstract}

\section{INTRODUCTION}

Wavefront sensors (WFS), which use solid-state CCD or CMOS photosensors, are one of the main sources of errors in adaptive optics systems. Inaccuracy in the detection of wavefront distortions introduces considerable errors into the wavefront reconstruction and leads to overall performance degradation of adaptive optics system. However, the simulation of photosensors for WFS, primarily solid-state CCD or CMOS photosensors, is a challenging task. $^{1,2}$ The main difficulty is that the photosensors is affected by many different sources of noise ${ }^{3}$ and nonlinearity (e.g., ADC non-linearity, $\mathrm{V} / \mathrm{V}$ and $\mathrm{V} / e^{-}$non-linearity) that cannot be modelled adequately using only Gaussian noise ${ }^{4}$ for performance estimation of the WFS.

Models of CMOS photosensors ${ }^{5-7}$ are often too specific $^{8,9}$ and describe complicated solid-state physical processes ${ }^{10}$ in photosensors. The models of CCD photosensors are generally simpler. ${ }^{11-14}$ For instance, the CCD camera noise model by $\mathrm{Cox}^{12}$ includes noises such as photon shot and electronic shot noise, dark-current noise, readout noise; however the model suggests that noise is localised and stationary. Healey and Kondepudy ${ }^{15}$ model camera noise with offset fixed-pattern noise (FPN), photon and dark-current shot noises, read noise and photo response non-uniformity (PRNU). A more complete model was formulated by Farrell et al ${ }^{5}$ with optics and noise sources including read-out noise, dark-current, offset FPN, PRNU and photon shot noise.

Despite considerable efforts in modelling of noise in CCD/CMOS sensors, results are often either incomplete or obtained using oversimplified assumptions. This work attempts to the issue of the lack of high-level photosensor modelling tools that enable adaptive optics engineers to simulate realistic effects of noise on the images received from the WFS.

In this paper, physical processes that cause noises are outlined and references are provided for further details. The Photon Transfer technique is used $^{16}$ for the simulation of the photosensor. Our model includes the light

Further author information: Mikhail V. Konnik: mail@mvkonnik.info, James Welsh: James.Welsh@newcastle.edu.au

Astronomical Adaptive Optics Systems and Applications IV, edited by Robert K. Tyson, Michael Hart, Proc. of SPIE Vol. 8149, 81490F · (C) 2011 SPIE · CCC code: 0277-786X/11/\$18 · doi: 10.1117/12.892667 
source and optical propagation with Fresnel approximation and models of noise sources: photon shot noise, FPN (several models), a read noise (dark current shot noise, dark pixel and column FPN, kTC, flicker noise, etc.). Procedures for characterisation of both light and dark noises of the simulated sensor based on the EMVA1288 standard $^{17}$ and other guidelines ${ }^{18,19}$ are sketched. An example of the simulated high-frame rate Shack-Hartmann wavefront sensor based on CMOS sensor is presented.

\section{PHOTONS TO CHARGE CONVERSION}

The ability of a semiconductor to produce electrons from incident photons is referred as quantum efficiency $Q E$. Quantum efficiency characterises the photon-to-photon efficiency from the pixel surface to the photosensor. In CMOS sensors, the QE is lower than that of a in CCD. In addition to lower internal QE, pixels in CMOS have a smaller photosensitive area that cause higher photon shot noise, higher current leakage, and more nonuniformities.

\subsection{Photon shot noise}

A process of photon capturing has an uncertainty that arises from random fluctuations when discrete photons are collected by the photodiode. Such uncertainty leads to photon shot noise and described by the Poisson probability distribution:

$$
p_{i}=\frac{P_{I}^{i}}{i !} e^{-P_{I}}
$$

where $p_{i}$ is the probability that there are $i$ interactions per pixel and $P_{I}$ is the number of interacting photons. In the case of a high level of the light signal (more then 1000 arrival events $^{20}$ of photons), Poisson distribution may be approximated by normal distribution.

\subsection{Gain Fixed Pattern Noise / Photo Response Non-Uniformity}

Gain fixed pattern noise* (FPN) is the spatial variation in pixel output values under uniform illumination. This is due to pixel-to-pixel variations in the geometry during fabrication of the sensor, ${ }^{3}$ variations in substrate material or interference with the local circuitry. Gain FPN increases with illumination and exponentially increases with

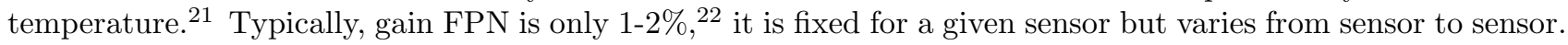
The percentage of the FPN with signal should be constant until reaching a full well for the photodiode.

\section{READ NOISE}

Read noise is defined ${ }^{16}$ as any noise that is not a function of the signal and generally consists of: dark current shot noise, dark offset FPN, sense node reset noise, source follower noise, ADC quantising noise. Thus, the general read noise equation, according to, ${ }^{16}$ is:

$$
\sigma_{R E A D}=\sqrt{\sigma_{D S H O T}^{2}+\sigma_{D F P N}^{2}+\sigma_{S N-R E S E T}^{2}+\sigma_{S F}^{2}+\sigma_{A D C}^{2}}
$$

Read noise components are described in the following subsections.

\subsection{Dark Current}

Even in the absence of light, pixels generate electrons that are referred as the dark current. The dark current is mostly due to thermal generation. The amount of dark charge varies from pixel to pixel and doubles with every $6-8^{\circ} \mathrm{C}$ increase of temperature. ${ }^{3}$ The average dark current rate can be characterised by $D_{R}\left[e^{-} / \mathrm{sec} / \mathrm{pixel}\right]$ and given by: ${ }^{22}$

$$
D_{R}=2.55 \cdot 10^{15} P_{A} D_{F M} T^{3 / 2} \exp \left[-\frac{E_{g a p}}{2 \cdot k T}\right],
$$

where $P_{A}$ is the pixel's area $\left[\mathrm{cm}^{2}\right], D_{F M}$ is the dark current figure-of-merit (varies significantly with sensor manufacturer) at $300 \mathrm{~K}\left[\mathrm{nA} / \mathrm{cm}^{2}\right], E_{\text {gap }}$ is the bandgap energy of the semiconductor which also varies with temperature, and $k$ is Boltzman's constant. Two forms of thermal dark current can be distinguished and simulated: dark current shot noise and offset FPN.

\footnotetext{
${ }^{*}$ Some authors refer to gain fixed pattern noise as photo response non-uniformity (PRNU).
} 


\subsection{Dark shot noise}

Additional shot noise from the dark current in the image sensor is called the dark current shot noise. ${ }^{22}$ The dark current shot noise is adequately described by the Poisson statistics as random arrival of the dark current electrons. Dark shot noise depends on the exposure time and the sensor temperature:

$$
\sigma_{D_{s h o t}}=\sqrt{D},
$$

where $D$ is the average dark current: ${ }^{16} D=t_{I} D_{R}$. Here $D_{R}$ is the average dark current rate $\left[e^{-} / \mathrm{sec} / \mathrm{pixel}\right]$ described above in Subsection 3.1.

\subsection{Offset Fixed Pattern Noise / Dark Signal Non-Uniformity}

Offset Fixed Pattern Noise (offset FPN) is a dark signal non-uniformity, which is the offset from the average dark current across the imaging array at a particular setting (temperature, integration time) in the absence of light. CMOS offset FPN is due to threshold differences in the pixel source follower amplifier, ${ }^{23}$ and can be column and pixel noise. ${ }^{24,25}$ Mathematically, offset FPN can be expressed ${ }^{16}$ as

$$
\sigma_{D_{F P N}}=D \cdot D_{N},
$$

where $D_{N}$ is the dark current FPN quality, which is typically between $10 \%$ and $40 \%$ for CCD and CMOS sensors, and $D=t_{I} D_{R}$. Here $D_{R}$ is the average dark current rate $\left[e^{-} / \mathrm{sec} / \mathrm{pixel}\right]$ described above in Subsection 3.1.

CMOS sensors typically have higher dark FPN than CCDs. CMOS sensors also suffer from pixel FPN and column FPN. Column FPN is caused by an offset in the integrating amplifier, size variations in the integrating capacitor, and also variation of bias/offset voltages. ${ }^{24}$ Column FPN appears as "stripes" in the image and can result in significant image quality degradation. CMOS sensors also exhibit pixel FPN as well as CCD sensors. The pixel FPN is due to variation in the photodetector geometry, dark current and due to variations in transistors parameters. There are several approaches for the simulation of pixel and column FPN in CMOS sensors.

\section{CHARGE TO VOLTAGE CONVERSION}

After the charge is generated in the pixel by the photo-effect, it is moved to the sense node to convert charge into voltage. The sense node is shared in the case of a CCD, whilst in CMOS sensors the sense node is located inside each pixel. The charge collected in each pixel of a sensor array is converted to voltage by z sense capacitor and a source-follower amplifier.

The simulation of the charge-to-voltage conversion can be performed according to Photon Transfer ${ }^{16}$ technique. One can use the sense node gain $A_{S N}$ as a parameter, which is typically $1 \ldots 5 \mu \mathrm{V} / \mathrm{e}^{-}$. Conversion from charge to voltage can then be made as follows:

$$
I_{V}=V_{R E F}+V_{\text {offset } F P N}+I_{e^{-}} \cdot A_{S N},
$$

where $V_{R E F}$ is the reference voltage, $V_{\text {offset FPN }}$ is the offset FPN, and $I_{e^{-}}$is the signal in electrons.

\subsection{Sense node Reset noise (kTC noise)}

Prior to the measurement of each pixel's charge packet, the sense capacitor is reset to a reference voltage level. ${ }^{26}$ Noise is generated at the sense node by an uncertainty in the reference voltage level due to thermal variations in the channel resistance of the MOSFET reset transistor. The reference level of the sense capacitor is therefore different from pixel to pixel. ${ }^{26}$ The reset noise -voltage is given by:

$$
\sigma_{R E S E T}\left(V_{S N}\right)=\sqrt{k T C_{S N} / q},
$$

where $\sigma_{R E S E T}\left(V_{S N}\right)$ is reset noise voltage [V], $R$ is the MOSFET channel resistance [Ohms], $k$ is Boltzmann's constant, the noise equivalent bandwidth $B=1 /(4 \tau)=1 /\left(4 R C_{S N}\right), q$ is a charge of an electron, and $T$ is the operation temperature $[\mathrm{K}]$. 
Sense node Reset noise: CCD case For CCD, the sense node reset noise is entirely removed by CDS.

Sense node Reset noise: CMOS case In CMOS photosensors, it is difficult to remove the reset noise for the specific CMOS pixels architectures even after application of CDS. The CDS suppresses the low frequency noise components, although increasing thermal noise contributions. ${ }^{27}$

\subsection{Source follower noise}

The source follower noise limits the read noise, however in high-end CCD and CMOS cameras the source follower noise has been driven down to one electron rms. ${ }^{16}$ Nonetheless, the source follower noise noticeably increases with an increasing frame rate. The source follower noise consists of white noise, flicker noise ( $1 / \mathrm{f}$ noise), and random telegraph noise (RTS).

\subsubsection{Johnson noise}

Similarly to the sense node reset noise (see Subsection 4.1), the source-follower amplifier has a resistance that generates a thermal noise, which is governed by the Johnson white noise equation. ${ }^{26}$ It is therefore either referred to as Johnson noise, ${ }^{28}$ Johnson-Nyquist, or simply as white noise. ${ }^{3}$

\subsubsection{Flicker $(1 / f)$ noise}

The flicker noise is commonly referred as $1 / f$ noise because of its approximate inverse dependence on frequency. For cameras in which pixels are read out at less than $1 \mathrm{MHz}$, the read noise floor is usually determined by $1 /$ f noise. $^{26}$ Sources of $1 / f$ noise in an image sensor are generated in the photo-diodes and the low-bandwidth analogue operation of MOS transistors due to imperfect contacts between two materials.

\subsubsection{Random Telegraph Signal (RTS) noise}

The low-frequency noise increases ${ }^{29}$ as the CCD and CMOS pixels shrink in dimension. In such devices, the low-frequency noise performance is dominated by Random Telegraph Signals (RTS) on top of the 1/f noise. The origin of such an RTS is attributed to the random trapping and emission of mobile charge carriers resulting in discrete modulation of the channel current.

\section{VOLTAGE TO DIGITAL NUMBERS CONVERSION}

After the sense node and the source follower, the data is digitised using an analogue to digital converter (ADC). In the simulations presented here, we use a simple linear model of an ADC. The resolution of an ADC is assumed as:

$$
K_{A D C}=\left(V_{\max }-V_{\min }\right) / N_{\max }
$$

where $V_{\max }$ is maximum voltage that can be quantified, $V_{\min }$ is minimum quantifiable voltage, and $N_{\max }=2^{M}$ is the number of voltage intervals ( $M$ is ADC's resolution in bits). The ADC's output can be represented as:

$$
A D C_{\text {Code }}=\operatorname{round}\left[\left(V_{\text {input }}-V_{\text {min }}\right) / K_{A D C}\right]
$$

The ADC noise is terms of noise electrons can be expressed as::

$$
\sigma_{A D C}=K_{A D C} / \sqrt{12}
$$

Furthermore we assume the ADC linear; however, the analogue to digital converters tends to have integral/differential non-linearity, which can influence on the data accuracy. Once can analyse the ADC non-linearity on photon transfer curve.

\section{SIMULATION METHODOLOGY}

The model of the photosensor for the Shack-Hartmann wavefront sensor was implemented using MATLABß in a modular fashion. Each module adds specific noise to the signal. Using the above-formulated noise models, one can therefore simulate CMOS or CCD sensors by turning on or off the appropriate modules. 


\subsection{Photon to Charge conversion}

The photon to electron conversion simulation is as follows. The light field is considered as a photon flux, and can be converted to photon quantities:

$$
Q_{p}=\frac{h \cdot c}{\lambda},
$$

where $Q_{p}$ is the energy of a photon at wavelength $\lambda$, Planck's constant $h$ and the speed of light $c$. Irradiance $I_{p h}$ of the light field is converted to [photons $\left./\left(\mathrm{m}^{2} \cdot \mathrm{sec}\right)\right]$ using Eq. 9. Next the signal in photons $I_{p h}$ is converted to a signal in electrons $I_{e^{-}}$:

$$
I_{e^{-}}=I_{p h} \cdot Q E \cdot P \cdot t_{I},
$$

where $Q E$ is quantum efficiency for visible and near-IR light, $P$ is the average number of incident photons per pixel, and $t_{I}$ is integration (exposure) time.

\subsubsection{Simulation of photon shot noise}

Photon shot noise can be optionally added on this stage. For the simulation of shot noise, a matrix is generated according to the number of photons in each pixel using the poissrnd MATLAB function. This matrix represents the image incorporating photon shot noise.

\subsubsection{Simulation of Gain FPN/ PRNU}

We use a Gaussian probability distribution to simulate Gain FPN/PRNU. This assumption is based on our experiments and estimation on hardware CMOS/CCD sensors.

\subsection{Read noise simulation}

The dark current is calculated using Eq.2 and is added to the signal as a constant. The value of the dark current is then used by other simulation modules (dark shot noise, dark FPN, etc.) as a mean value for the noise.

\subsubsection{Simulation of dark shot noise}

The dark shot noise is simulated using the poissrnd function in MATLAB. We use the value of the pixel as the input of poissrnd, and on the output we have a signal with added uncertainty that is Poisson distributed random variable.

\subsubsection{Simulation of offset FPN / DSNU}

Simulation of the offset FPN is more difficult since for CCD and CMOS the noise is significantly different. Offset FPN for CCD image sensors is random. CCD sensors suffer only from pixel FPN due to spatial variation in the photodetector geometry and dark current. CMOS sensors typically have higher FPN than CCDs, and CMOS sensors suffer from both pixel FPN and column FPN.

Simulation of dark FPN in CCD. The dark FPN is modelled as a sample from a spatial white noise process $^{24}$ in a CCD image sensor. This is justified by the fact that in a typical CCD sensor all pixels share the same output amplifier. For the dark FPN simulation of CCD sensors, with low dark current, it was reported ${ }^{30}$ that the Gamma distribution is the most appropriate.

Simulation of dark FPN in CMOS. Several methods of simulation of dark FPN can be considered. A statistical model for FPN in CMOS sensors was introduced in. ${ }^{24}$ The FPN is represented as the sum of two components: a column and a pixel component. Each component is modelled by a first order isotropic autoregressive process, and the processes are assumed uncorrelated. The advantage of such a model is that parameters can be estimated easily and efficiently from the data of a hardware sensor. ${ }^{31}$

Another concept of offset FPN modelling is reported in. ${ }^{2}$ The proposed concept is to superimpose two distributions in order to achieve a more realistic noise distribution. The first distribution describes the main body of dark FPN and then a uniform distribution is superimposed to model "leaky" pixels. There are other reports ${ }^{30}$ that the Log-Normal distribution works well for conventional 3T APS CMOS sensors with comparatively high dark current. 


\subsubsection{Simulation of sense node reset noise / kTC noise}

For the simulation of sense node reset noise, the notation of sense node gain is mode convenient:

$$
\sigma_{R E S E T}\left(V_{S N}\right)=\sqrt{k T / A_{S N}},
$$

where $A_{S N}$ is a sense node gain $\left(\mu V / e^{-}\right)$. Because reset noise can be significant ${ }^{26}$ (about $50 \mathrm{rms}$ electrons), most high-performance cameras incorporate a mechanism to eliminate it, for instance, correlated double sampling (CDS). CDS is used in many analogue circuits to reduce offsets and reset noise. The idea is to sample the output twice: once right after reset and the second time with the signal present. In this way the offset and reset noise are eliminated by taking the difference. ${ }^{32}$

\subsubsection{Simulation of source follower noise}

The power spectrum of the source follower noise can be described ${ }^{16}$ as:

$$
S_{S F}(f)=W(f)^{2} \cdot\left(1+\frac{f_{c}}{f}\right)+S_{R T S}(f),
$$

where $W(f)$ is the thermal white noise $\left[V / H z^{1 / 2}\right.$, typically $\left.15 n V / H z^{1 / 2}\right]$, flicker noise corner ${ }^{\dagger}$ frequency $f_{c}$ in $[\mathrm{Hz}]$. The RTS power spectrum is given ${ }^{16}$ by:

$$
S_{R T S}(f)=\frac{2 \Delta I^{2} \tau_{R T S}}{4+\left(2 \pi f \tau_{R T S}\right)^{2}},
$$

where $\tau_{R T S}$ is the RTS characteristic time constant [sec] and $\Delta I$ is the source follower current modulation induced by RTS [A]. The source follower noise can be approximated as:

$$
\sigma_{S F}=\frac{\sqrt{\int_{0}^{\infty} S_{S F}(f) H_{C D S}(f) d f}}{A_{S N} A_{S F}\left(1-\exp ^{\left.-t_{s} / \tau_{D}\right)}\right.}
$$

where $\sigma_{S F}$ is the source follower noise $\left[e^{-} \mathrm{rms}\right], f$ is the electrical frequency [Hz], $t_{s}$ is the CDS sample-tosampling time [sec], $\tau_{D}$ is the CDS dominant time constant, which is usually ${ }^{22}$ set to $\tau_{D}=0.5 t_{s}$ [sec]. The $H_{C D S}(f)$ function is the CDS transfer function is: ${ }^{16}$

$$
H_{C D S}(f)=\frac{1}{2-2 \cos \left(2 \pi f t_{s}\right)} \cdot\left[1+\left(2 \pi f \tau_{D}\right)^{2}\right]
$$

Here the first term sets the CDS bandwidth for the white noise rejection before sampling takes place through $B=1 /\left(4 \tau_{D}\right)$, where $B$ is defined as the noise equivalent bandwidth $[\mathrm{Hz}]$.

In CCD photosensors, source follower noise is typically limited by the flicker noise.

In CMOS photosensors, source follower noise is typically limited by the RTS noise. ${ }^{16}$

\section{NUMERICAL SIMULATION OF THE CMOS PHOTOSENSOR IN THE SHACK-HARTMANN WAVEFRONT SENSOR}

The model of a $1024 \times 1024$ pixel CMOS photosensor was implemented in MATLAB $\AA$ using the modular approach described above. The sensor was simulated using:

1. photon shot noise and PRNU for light noises (see Section 2);

2. dark current, dark shot noise and offset FPN (dark FPN) as described in Section 3

\footnotetext{
${ }^{\dagger}$ Flicker noise corner frequency is the frequency where the power spectrum of white and flicker noise are equal.
} 
3. sense node reset noise and source follower noise (see Section4).

The photosensor was considered linear, i.e. non-linearity such as ADC, $\mathrm{V} / \mathrm{V}$ and $\mathrm{V} / e^{-}$were not applied to the signal. The main parameters of the simulated photosensor for typical Shack-Hartmann wavefront sensor are summarised in Table 1.

Table 1. Parameters of simulated CMOS sensor for SH WFS.

\begin{tabular}{cc}
\hline \hline Sensor's parameter & Value \\
\hline \hline wavelength $\lambda$ & $0.55 \mu m$ \\
\hline pixel size & $5.00 \mu m$ \\
\hline pixel fill factor & $50 \%$ \\
\hline Full well & $20000 e^{-}$ \\
\hline QE & 0.80 \\
\hline PRNU / FPN factor & $1 \%$ \\
\hline dark FPN factor & $10 \%$ \\
\hline Column FPN factor & $0.10 \%$ \\
\hline Temperature & $300 \mathrm{~K}$ \\
\hline dark current figure of merit & $1.00 \mathrm{nA} / \mathrm{cm}^{2}$ \\
\hline Sense node gain & $5.00 \mu \mathrm{V} / \mathrm{e}^{-}$ \\
\hline Clock speed & $20 \mathrm{MHz}$ \\
\hline ADC bit & $10 \mathrm{bit}$ \\
\hline
\end{tabular}

The realistic light fields for the numerical simulations were generated using the simulated Lambertian light source. 33 The classical case "Object against the lens" 34 was used for the simulation of lenslets in the ShackHartmann WFS. The lenslets were assumed free from optical defects.

The results below were obtained using the simulated CMOS photosensor with parameters summarised in Table 1. The assumed frame rate of the simulated photosensor was 1000 frames per second (affects the source follower noise, see Subsection 4.2). The radiometric function, the noise spectrogram, the signal-to-noise ratio, and the dependency of signal versus noise were estimated. Parameters such as PRNU and FPN ratio were evaluated from the measurements.

\subsection{Radiometric function of the simulated photosensor}

The radiometric function has been measured in order to confirm that the response of the simulated CMOS photosensor is linear. We simulated the flat-field scenes with different integration times and "registered" those scenes by the simulated photosensor. The integration time of the captured images was varied from $1 \cdot 10^{-4}$ up to 1 sec., where a saturation occurs. Four images were taken and averaged for each integration time. For each flat-field image, a $512 \times 512$ pixels area from the centre of the averaged image was used. The mean value of the $512 \times 512$ pixels area forms the radiometric curve (see Fig. 1), and the standard deviation indicates the uncertainty of the measurements. One can see from the Fig. 1 that the simulated sensor is highly linear as expected.

\subsection{Photon Transfer Curve for the simulated sensor}

The Photon Transfer Curve (PTC) was measured using the following procedure. The scene that covers the dynamic range was simulated. Such a scene can be visually described as a linear gradient. There were 64 frames of such scene taken and averaged. Two arrays were evaluated from the averaged data: the mean values of pixels $S_{\text {mean }}$ and the standard deviations of pixels $S_{s t d}$. Furthermore, the values of the signal were picked from the array $S_{\text {mean }}$ with a step of 1 DN. For each signal's value in $S_{\text {mean }}$, the corresponding standard deviation value from the array $S_{s t d}$ was found as an estimation of noise. ${ }^{35}$ 


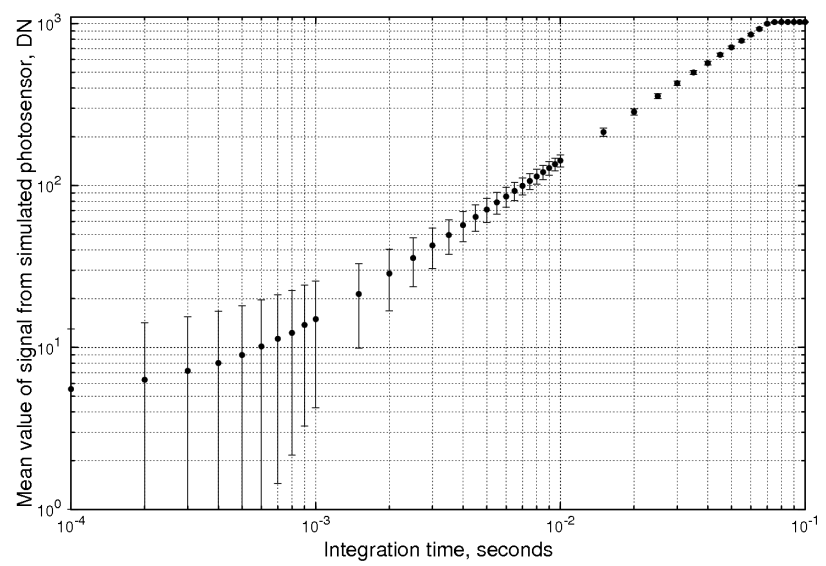

Figure 1. The radiometric function of the simulated CMOS photosensor.

Simulation results The resulting Photon Transfer Curve (PTC) plot is presented in Fig. 2a for various noise cases.

First, only the dark current shot noise was turned on; one can see the distinct slope of the PTC curve in Fig. 2a (marked by "+").

In the second experiment we turned on shot noise, dark current and FPN (PRNU) with PRNU factor 0.01 as in Table 1. The slope of PTC changed because the FPN is directly proportional to the signal, which is consistent with. $^{18}$

The dark pixel FPN noise of factor 0.1 was added in the third experiment to the noise picture using the autoregressive model. As a result, the PTC was altered in the low-light region due to the additional FPN noise, as seen in Fig. 2a marked by "o" symbol.

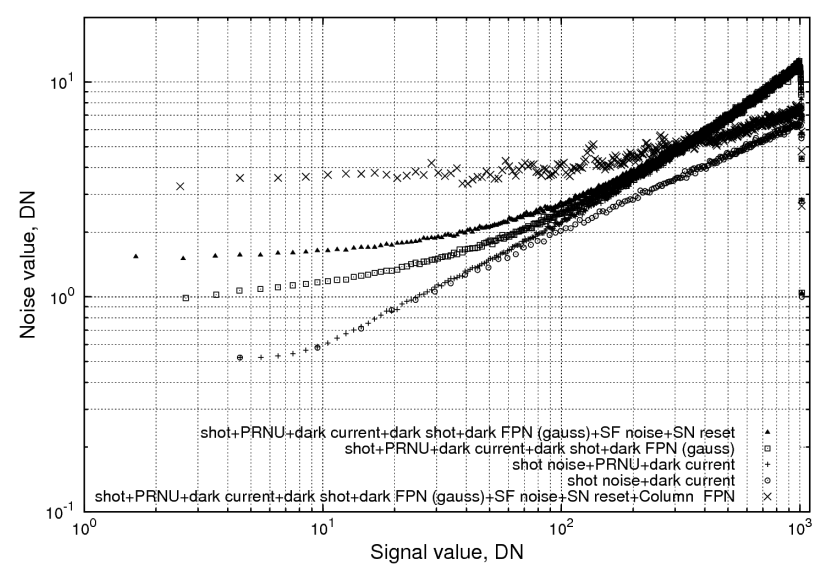

a)

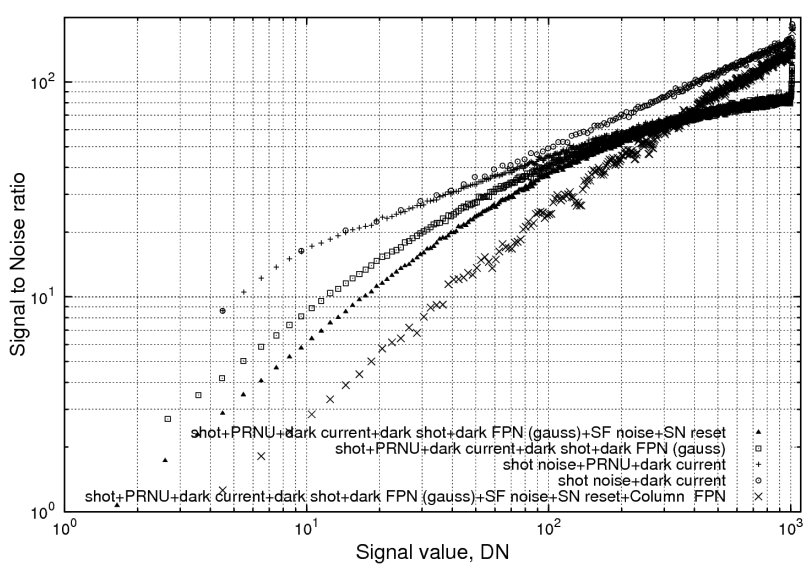

b)

Figure 2. The results for the simulated CMOS photosensor: a) the Photon Transfer Curve, and b) the signal to noise ratio versus signal.

A fourth experiment added the sense node reset noise (see Section 4.1) and the source follower noise (see Section 4.2) to the noise sources in the third experiment. One can see from the "॥" marked PTC in Fig. 2a that the overall noise has been increased in the low-light section even more.

Finally, we added the column FPN offset noise (marked with symbol " $\times$ " on the plots) that was of factor $0.1 \%$ from the reference voltage $V_{R E F}$. It is difficult to see on the image by visual inspection; as such noise severely degrades signal and SNR performance. 


\subsection{Signal-to-noise performance}

From the theoretical standpoint, the signal-to-noise ratio (SNR) as a function of signal plotted on a log-log scale, must exhibit three different regimes. ${ }^{16}$ The shot noise regime occurs in intermediate-light conditions producing the slope $1 / 2$ on the plot. Then the FPN regime begins to emerge in the middle and high levels of signal with zero slope on the SNR plot. Finally, the full well regime occurs at the saturation levels.

Simulation results The SNR dependence for the several of noise combinations has been calculated during the experiments with Photon Transfer Curve (see Subsection 7.2 above). The results of the simulations are presented in Fig.2b. A consistent behaviour with theory is observed.

\subsection{Noise spectrograms}

The measurement approach of the noise spectrogram described in this section is based on the "Spectrogram Method". ${ }^{17}$ The total noise is taken from a spectrogram of a single image. The spectrogram is computed by taking the mean of the amplitude of the FFT on each line. First, the mean value of the image is computed as:

$$
\mu_{y}=(1 / M N) \cdot \sum_{n} \sum_{m} y(n, m) .
$$

Then for the $j$-th of the $M$ lines of the image, the amplitude of the FFT is computed. Next, the amplitude $S_{j}$ of the FFT:

$$
S(n)=\sqrt{(1 / M) \cdot \sum S_{j}(n)^{2}}
$$

is computed for the $j$-th line. The $N+1$ values $S(n)$ with $0 \leq n \leq N$ form the spectrogram of the image.

The mean of the squared transform is the variance of the noise, describing the total grey value noise, including all artefacts. It is computed as: $\sigma_{y \text {.full }}^{2}=[1 /(N+1)] \cdot \sum_{n} S(n)^{2}$. The square of the height of the flat section seen in the spectrogram curve is the variance describing the white part of the noise. It is estimated by taking the median of the spectrogram. As seen from the spectrograms, the read noise can be considered as white. The non-whiteness ${ }^{17}$ coefficient was calculated as: $F=\sigma_{y . f u l l}^{2} / \sigma_{y . w h i t e}^{2}$ and should be $F \approx 1$.

Simulation results First, read noise spectrograms for different integration times were estimated. For the $t_{I_{1}}=5.0 \cdot 10^{-4} \mathrm{sec}$ non-whiteness coefficient was $F=1.0016 \pm 0.0018$, for $t_{I_{2}}=5.0 \cdot 10^{-2} \mathrm{sec} F=1.0029 \pm 0.0018$, and for $t_{I_{3}}=5.0 \mathrm{sec} F=1.0046 \pm 0.0018$ is in excellent consistency with the model. ${ }^{17}$ Uncertainness of the non-whiteness coefficient $F$ was estimated from the standard deviation of 100 measurements of $F$ (uncertainty is constant regardless of integration time).

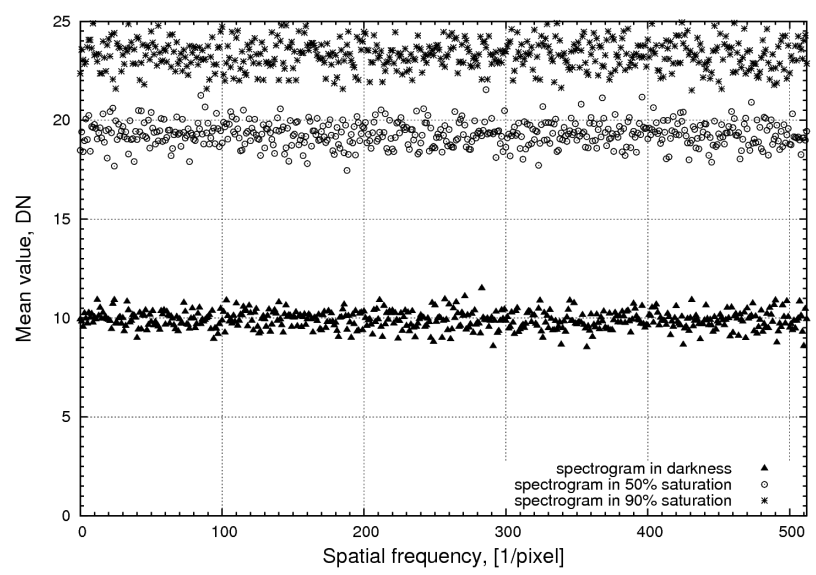

Figure 3. Three noise spectrograms: in darkness, at 50\% light saturation, and at $90 \%$ light saturation. 
Three spectrograms were calculated in order to estimate the noise statistics. One spectrogram was simulated in darkness with the integration time $t_{I_{1}}=5.0 \cdot 10^{-2} \sec$ (non-whiteness coefficient was estimated $F=0.9995 \pm$ 0.0017). The second spectrogram was simulated with the illumination turned on and the integration time set such that the sensor is at $50 \%$ saturation with $t_{I_{2}}=3.5 \cdot 10^{-2} \mathrm{sec}$ and estimated as $F=0.9993 \pm 0.0017$. Finally, the third spectrogram was simulated with the integration time set to give $90 \%$ saturation with $t_{I_{1}}=6.5 \cdot 10^{-2} \mathrm{sec}$ and the estimated $F=1.0002 \pm 0.0017$. Three spectrograms, according to EMVA1288 standard, ${ }^{17}$ were drawn in one diagram (see Fig. 3) showing $S(n) / K \eta$ in [p ] versus the spatial frequency [1/pixel]. According to the model, ${ }^{17}$ spectrograms should be flat with occasional peaks only. The results of the noise spectrogram for the simulated sensor in Fig. 3 are consistent with the model. ${ }^{17}$

\subsection{Dark noise performance}

The dark current parameters and the resulting dark current can be characterised by plotting dark noise as a function of the dark current signal. The Photon Transfer Curve (PTC) generated without a light source is referred to as a Dark Transfer Curve (DTC). The DTC can provide insights on the noise picture of a photosensor.

Simulation results In order to measure the Dark Transfer Curve, we switched off the virtual light and conducted measurements in darkness by varying the integration time of the simulated photosensor. The comparison between the simulation of dark FPN using the autoregressive model and the Wald distribution is provided. The results of the DTC simulation are presented in Fig. 4 for various noise cases.

First, only the dark current was turned on. As expected, the constant noise level increases with the integration time according to Eq. 2. In the second experiment, we turned on the dark shot noise. The resulting DTC is of slope 1/2 as shown in Fig. 4 with data marked by "•" symbol.

The third experiment consisted of the dark current, the dark shot noise, and the dark pixel FPN. For the dark pixel FPN, we used Wald distribution combined with uniform distribution in order to simulate the dark FPN in CMOS sensors (especially non-cooled and on relatively long integration time). The dark pixel FPN was added with the dark noise factor of $0.1 \%$ and the Wald distribution parameter $\lambda_{W a l d}=2$. The resulting DTC in Fig. 2 is marked by the " $\nabla$ " symbol. The slope of DTC has changed as compared with dark shot noise that is consistent with theoretical assumptions. ${ }^{16}$

The forth experiment changed the dark pixel FPN noise to an autoregressive model of factor 0.1. As a result, it altered the noise levels, as seen as "ム" marked DTC in Fig. 2.

In the fifth experiment, we added sense node reset noise and source follower noise to the existing noise sources in the third experiment. One can see from the "Ш" marked DTC in Fig. 4 that the overall noise has been increased in the low-light part.

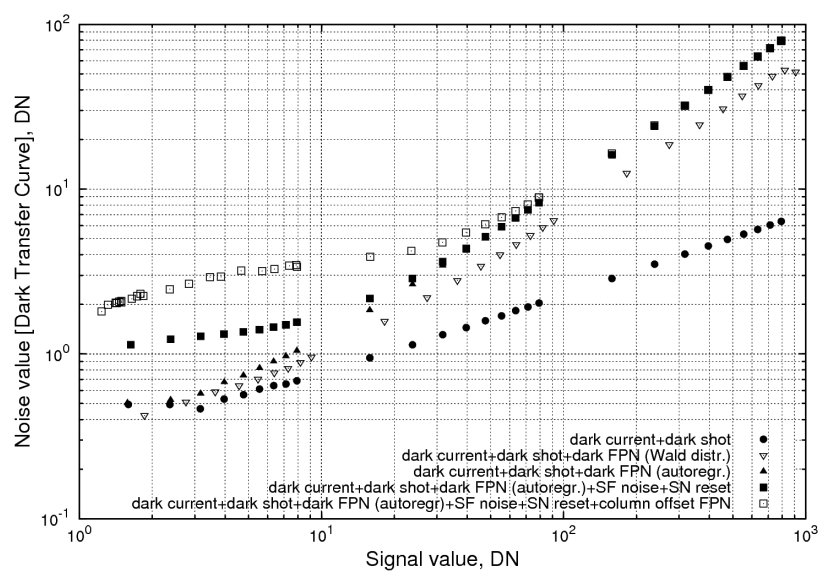

Figure 4. Dark Transfer Curve for the simulated photosensor. 
Finally, the column FPN offset noise was added (marked with " $\square$ " symbol on the plot) that was of the factor $0.1 \%$ from the reference voltage $V_{R E F}$. As before in PTC experiments, column offset FPN noise severely degrades signal and SNR performance. The behaviour of the noise is evidently different from the other noises, as seen in Fig. 4.

\section{CONCLUSION}

Accuracy of wavefront sensors used in adaptive optics is affected by noise in CCD or CMOS photosensors. Since most wavefront sensors use CCD or CMOS sensors, it is necessary to use an accurate model of the photosensor in numerical simulations. However, the model of photosensors is often based on oversimplified assumptions leading to inaccurate results of performance estimations of wavefront sensors. Hence, it is important to formulate a high-level model of photosensor that will enable engineers to simulate realistic effects of noise from wavefront sensors.

Using the formulated high-level model, a $1024 \times 1024$ pixels CMOS photosensor (which are commonly used in wavefront sensors) with a 10 bit ADC has been simulated. The results of simulation of the Photon Transfer curves (PTC) are consistent with theory and reveal the impact of specific noise sources to the whole noise picture. The spectrogram method has been used to show that the spectrum of the read noise as well as signal tends to be flat. The non-whiteness coefficient was calculated for three cases of the simulation and is $F \approx 1$, which is consistent with the model of the EMVA1288 standard. Numerical simulations and the comparison with a real CMOS sensor allow us to say that in case of CMOS sensors, skewed probability distributions are more adequate for description of dark FPN.

The results of numerical experiments are in good agreement with theory. The results reveal the difference between certain models of noise and provide an estimation of noise influence on the images quality. The formulated high-level model can be transformed from the simulation of CMOS photosensors to CCD. In this regard the model is flexible enough to be used for simulation either wavefront sensors or the scientific sensors for the astronomical objects registration.

\section{ACKNOWLEDGEMENTS}

The authors are gratefully acknowledge personal communications with James $R$. Janesick and his valuable comments.

\section{REFERENCES}

[1] Irie, K., McKinnon, A., Unsworth, K., and Woodhead, I., "A model for measurement of noise in CCD digital-video cameras," Measurement Science and Technology 19, 045207 (2008).

[2] Gow, R., Renshaw, D., Findlater, K., Grant, L., McLeod, S., Hart, J., and Nicol, R., "A Comprehensive Tool for Modeling CMOS Image-Sensor-Noise Performance," IEEE Transactions on Electron Devices 54(6), 1321-1329 (2007).

[3] Hornsey, R., [Part III: Noise in Image Sensors], University of Waterloo (1999).

[4] Jiang, Z., Gong, S., and Dai, Y., "Monte-carlo analysis of centroid detected accuracy for wavefront sensor," Optics 83 Laser Technology 37(7), 541 - 546 (2005).

[5] Farrell, J., Xiao, F., Catrysse, P., and Wandell, B., "A simulation tool for evaluating digital camera image quality," in [Proc. SPIE], 5294, 124-131 (2004).

[6] Kolehmainen, T., Aikio, J., Karppinen, M., Mattila, A., Makinen, J., Kataja, K., Tukkiniemi, K., and Karioja, P., "Simulation of imaging system's performance," in [Proceedings of SPIE], 5178, 204 (2004).

[7] Wach, H. and Dowski Jr, E., "Noise modeling for design and simulation of computational imaging systems," in $[$ Proceedings of SPIE], 5438, 159 (2004).

[8] Tian, H. and El Gamal, A., "Analysis of 1/f noise in CMOS APS," in [Proc. SPIE], 3965, 168-175 (2000).

[9] Lee, J. S., Hornsey, R., and Renshaw, D., "Analysis of cmos photodiodes. i. quantum efficiency," IEEE Transactions on Electron Devices 50, 1233 - 1238 (May 2003). 
[10] Shcherback, I., Danov, T., and Yadid-Pecht, O., "A comprehensive cmos aps crosstalk study: photoresponse model, technology, and design trends," IEEE Transactions on Electron Devices 51(12), 2033 - 2041 (2004).

[11] Li, H., Song, H., Rao, C., and Rao, X., "Accuracy analysis of centroid calculated by a modified center detection algorithm for shack-hartmann wavefront sensor," Optics Communications 281(4), 750 - 755 (2008).

[12] Boie, R. and Cox, I., "An analysis of camera noise," IEEE Transactions on Pattern Analysis and Machine Intelligence 14, 671-674 (Jun 1992).

[13] Costantini, R. and Süsstrunk, S., "Virtual sensor design," Proc. ISEST/SPIE Electronic Imaging 2004: Sensors and Camera Systems for Scientific, Industrial, and Digital Photography Applications 5301, 408419 (2004).

[14] Jiang, W., Xian, H., and Shen, F., "Detecting error of Shack-Hartmann wavefront sensor," in [Proceedings of SPIE, Adaptive optics and applications], 534-544 (1997).

[15] Healey, G. and Kondepudy, R., "Radiometric ccd camera calibration and noise estimation," IEEE Transactions on Pattern Analysis and Machine Intelligence 16(3), 267-276 (1994).

[16] Janesick, J. R., [Photon Transfer], SPIE International Society for Optical Engineering (2007).

[17] EMVA STANDARD 1288, "Standard for measurement and presentation of specifications for machine vision sensors and cameras," Release A2.01 (2005).

[18] James R. Janesick, "CCD characterization using the photon transfer technique," in [Proc. SPIE, Solid State Imaging Arrays, Vol.570, pages 7-19,], (1985).

[19] PixeLINK, "How to interpret camera parameters," http://www.pixelink.com/ web publication (2007).

[20] Mendenhall, W. and Sincich, T., [Statistics for Engineering and the Sciences], Prentice-Hall, Inc. Upper Saddle River, NJ, USA (2006).

[21] Nakamura, J., [Image sensors and signal processing for digital still cameras], CRC Press (2006).

[22] James R. Janesick, [Scientific Charge-Coupled Devices], SPIE PRESS Monograph (2001).

[23] Janesick, J. R., "personal communication," (2011).

[24] El Gamal, A., Fowler, B., Min, H., and Liu, X., "Modeling and estimation of FPN components in CMOS image sensors," in [Proc. SPIE], 3301, 168-177 (1998).

[25] Kelly, S., Guidash, R., and Pillman, B., "Fixed pattern noise removal in CMOS imagers across various operational conditions," (Oct. 28 2008). US Patent 7,443,431.

[26] Fellers, T. J. and Davidson, M. W., "CCD noise sources and signal-to-noise ratio," tech. rep., National High Magnetic Field Laboratory, The Florida State University, Tallahassee, Florida, USA. (2010).

[27] Deptuch, G., Berst, J., Claus, G., Colledani, C., Dulinski, W., Goerlach, U., Gornoushkin, Y., Hu, Y., Husson, D., Orazi, G., Turchetta, R., Riester, J., and Winter, M., "Design and testing of monolithic active pixel sensors for charged particle tracking," in [IEEE Nuclear Science Symposium Conference Record, volume 1, pages 3/103 -3/110], (2000).

[28] Johnson, J. B., "Thermal agitation of electricity in conductors," Phys. Rev. 32, 97 (Jul 1928).

[29] Kolhatkar, J., Vandamme, L., Salm, C., and Wallinga, H., "Separation of random telegraph signals from 1/f noise in MOSFETs under constant and switched bias conditions," in [European Solid-State Device Research, 2003 33rd Conference on. ESSDERC'03], 549-552, IEEE (2004).

[30] Baer, R. L., "A model for dark current characterization and simulation," in [Electronic Imaging Conference, SPIE Vol. 6068, page 37-48], 6068, 37-48 (2006).

[31] Yang, D., Gamal, A., Fowler, B., and Tian, H., "A 640× 512 CMOS image sensor with ultrawide dynamic range floating-point pixel-level ADC," IEEE Journal of Solid-State Circuits 34(12), 1821-1834 (2002).

[32] Varidel, D., Bourquin, J.-P., Bovet, D., Fiorucci, G., and Schenker, D., "CCDs as low-energy x-ray detectors : II. technical aspects," Nuclear Instruments and Methods in Physics Research, Section A: Accelerators, Spectrometers, Detectors and Associated Equipment 292(1), 147 - 155 (1990).

[33] Schmidt, J. D., [Numerical Simulation of Optical Wave Propagation, With Examples in Matlab], Society of Photo-Optical Instrumentation Engineers (2010).

[34] Goodman, J. W., [Introduction to Fourier Optics], McGraw-Hill book company (1968).

[35] Konnik, M., Manykin, E., and Starikov, S., "Extension of the possibilities of a commercial digital camera in detecting spatial intensity distribution of laser radiation," Quantum Electronics 40, 314-320 (2010). 